\title{
Some Fixed Points Results of Quadratic Functions in Split Quaternions
}

\author{
Young Chel Kwun, ${ }^{1}$ Mobeen Munir, ${ }^{2}$ Waqas Nazeer, ${ }^{2}$ and Shin Min Kang $^{3}$ \\ ${ }^{1}$ Department of Mathematics, Dong-A University, Busan 49315, Republic of Korea \\ ${ }^{2}$ Division of Science and Technology, University of Education, Lahore 54000, Pakistan \\ ${ }^{3}$ Department of Mathematics, Gyeongsang National University, Jinju 52828, Republic of Korea
}

Correspondence should be addressed to Waqas Nazeer; nazeer.waqas@ue.edu.pk and Shin Min Kang; smkang@gnu.ac.kr

Received 2 May 2016; Accepted 4 July 2016

Academic Editor: Pasquale Vetro

Copyright (C) 2016 Young Chel Kwun et al. This is an open access article distributed under the Creative Commons Attribution License, which permits unrestricted use, distribution, and reproduction in any medium, provided the original work is properly cited.

We attempt to find fixed points of a general quadratic polynomial in the algebra of split quaternion. In some cases, we characterize fixed points in terms of the coefficients of these polynomials and also give the cardinality of these points. As a consequence, we give some simple examples to strengthen the infinitude of these points in these cases. We also find the roots of quadratic polynomials as simple consequences.

\section{Introduction}

Because of the ever increasing interests in higher dimension spaces and algebras, mathematicians and physicists are in search for developing an easy understanding towards them so that they better understand the physical phenomenons. In an attempt to satisfy this curiosity, they consider different functions to analyze them and also discuss some fixed points of these functions see $[1,2]$. These algebras may present nice understanding towards general rotations and describe some easy ways to consider geometric problems and also problems in mechanics and dynamical systems [3-7].

This paper mainly covers split quaternion algebra or coquaternion over real numbers. It was in fact introduced by James Cockle in 1849 on already established quaternions by Hamilton in 1843. Both of these algebras are actually associative but noncommutative and non-division ring generated by four basic elements. Like quaternion, split quaternion also forms a four-dimensional real vector space equipped with a multiplicative operation. But, unlike the quaternion algebra, the split quaternion algebra contains lots of zero divisors, nilpotents, and nontrivial idempotents. For a detailed description of quaternion and its generalization (octonion), see [3, 8-10]. As mathematical structures, both are algebras over the real numbers which are isomorphic to the algebra of $2 \times 2$ real matrices. The name split quaternion is used due to the division into positive and negative terms in the modulus function. The set $(1, i \cdot j, k)$ forms a basis. The products of these elements are

$$
\begin{aligned}
& i^{2}=-1, \\
& j^{2}=1=k^{2}, \\
& i j=k=-j i, \\
& j k=-i=-k j, \\
& k i=j=-i k, \\
& i j k=1 .
\end{aligned}
$$

It follows from the defining relations that the set $( \pm 1, \pm i$, $\pm j, \pm k)$ is a group under split quaternion multiplication; it is 
isomorphic to the dihedral group of a square. Equation (2) encodes the multiplication of basis split quaternions:

\begin{tabular}{|c|c|c|c|c|}
\hline$\cdot$ & 1 & $i$ & $j$ & $k$ \\
\hline 1 & 1 & $i$ & $j$ & $k$ \\
\hline$i$ & $i$ & -1 & $k$ & $-j$ \\
\hline$j$ & $j$ & $-k$ & 1 & $-i$ \\
\hline$k$ & $k$ & $j$ & $i$ & 1 \\
\hline
\end{tabular}

Both of these algebras have a long history and a great deal of literature is at hand on them. Brand in [4] gave the roots of a quaternion. Strictly speaking, he proved mainly De Moivre's theorem and then used it to find $n$th roots of a quaternion. His approach paved the way for finding roots of a quaternion in an efficient and intelligent way. Recently, authors in [1] considered split quaternionic matrices and discussed their interesting properties. Erdoğdu and Özdemir computed the eigenvalues for these matrices [5]. Ozdemir also discussed the roots of split quaternions in [2]. He also gave some geometrical applications of split quaternions in [6]. Rotations in Minkowski 3-space can be explained easily with split quaternions, such as Euclidean rotations used with quaternions [7]. So, it is very much possible to perform many things with split quaternions which can ordinarily be done using vectors analysis with Lorentzian metric and vector products. It is important to mention that these two algebras can also be constructed for $\mathbb{Z}_{p}$ over prime finite fields of characteristic $P$. Then, we obtain finite families of algebras with entirely different properties. For these finite algebras, see [11].

In the present paper, we first obtain the roots of a general quadratic polynomial in algebra of split quaternion. As simple implications, we state that there are infinitely many roots for some special cases. We also associate some general functions with these polynomials and explore their fixed points. In the end, we give some nice examples for each case. First, we make a preliminary introduction which will be used for the rest of this paper.

Definition 1. Let $x \in \mathbb{\mathbb { H } _ { s }}$; then, $x$ has the form $x=a_{0}+a_{1} i+$ $a_{2} j+a_{3} k$, where $a_{i} \in \mathbb{R}$. The conjugate of $x$ is defined as $x=$ $a_{0}-a_{1} i-a_{2} j-a_{3} k$. The norm of $x$ is given by

$$
N(x)=a_{0}^{2}+a_{1}^{2}-a_{3}^{2}-a_{4}^{2} .
$$

We are first interested in explicit formulas for computing the roots of a quadratic polynomial of the form $x^{2}+b x+c$, where $b, c \in H_{s}$. Let us fix $x=x_{0}+x_{1} i+x_{2} j+x_{3} k, b=$ $b_{0}+b_{1} i+b_{2} j+b_{3} k$, and $c=c_{0}+c_{1} i+c_{2} j+c_{3} k$. So, $x^{2}+b x+c=0$ becomes the real system of nonlinear equations

$$
\begin{aligned}
& \left(x_{0}+x_{1} i+x_{2} j+x_{3} k\right)^{2} \\
& \quad+\left(b_{0}+b_{1} i+b_{2} j+b_{3} k\right)\left(x_{0}+x_{1} i+x_{2} j+x_{3} k\right)+c_{0} \\
& \quad+c_{1} i+c_{2} j+c_{3} k=0,
\end{aligned}
$$

$$
\begin{aligned}
& x_{0}^{2}-x_{1}^{2}+x_{2}^{2}+x_{3}^{2}+b_{0} x_{0}-b_{1} x_{1}+b_{2} x_{2}+b_{3} x_{3} \\
& \quad+c_{0}=0, \\
& 2 x_{0} x_{1}+b_{1} x_{0}+b_{0} x_{1}+b_{3} x_{2}-b_{2} x_{3}+c_{1}=0, \\
& 2 x_{0} x_{2}+b_{2} x_{0}+b_{3} x_{1}+b_{0} x_{2}-b_{1} x_{3}+c_{2}=0, \\
& 2 x_{0} x_{3}+b_{3} x_{0}+b_{1} x_{2}+b_{0} x_{3}-b_{2} x_{1}+c_{3}=0 .
\end{aligned}
$$

We obtain five equations, and by no means is it obvious that this nonlinear system will have an explicit solution. There have been several attempts in the literature for the case of quaternion. Historically speaking, split quaternion preceded Cayley's matrix algebra; split quaternion (along with quaternion and tessarines) evokes the broader Linear Algebra.

\section{Main Results}

We first solve a general quadratic polynomial in the algebra of split quaternion. As a consequence, we find fixed points of associated functions in this algebra.

Proposition 2. $x=(-b / 2+\beta i+\gamma j+\delta k)$ is a root of $x^{2}+b x+c=$ 0 with $b^{2}<4 c$ and $b, c \in \mathbb{R}$, where $\beta^{2}-\gamma^{2}-\delta^{2}=\left(4 c-b^{2}\right) / 4$ and $\beta, \gamma, \delta \in \mathbb{R}$.

Proof. Take $x=\alpha+\beta i+\gamma j+\delta k$ in $x^{2}+b x+c=0$ given that $b, c \in \mathbb{R}$ and $b^{2}<4 c$.

We get $(\alpha+\beta i+\gamma j+\delta k)^{2}+b(\alpha+\beta i+\gamma j+\delta k)+c=0$. Consider

$$
\begin{aligned}
& \alpha^{2}-\beta^{2}+\gamma^{2}+\delta^{2}+2 \alpha \beta i+2 \alpha \gamma j+2 \alpha \delta k+b \alpha+b \beta i \\
& \quad+b \gamma j+b \delta k+c=0 \\
& \alpha^{2}-\beta^{2}+\gamma^{2}+\delta^{2}+b \alpha+c=0 \\
& 2 \alpha \beta+b \beta=0 \\
& 2 \alpha \gamma+b \gamma=0 \\
& 2 \alpha \delta+b \delta=0
\end{aligned}
$$$$
\beta \neq 0
$$$$
\text { so } 2 \alpha+b=0 \Longrightarrow \alpha=-\frac{b}{2} \text {; }
$$

put $\alpha=-b / 2$; consider

$$
\begin{aligned}
\left(-\frac{b}{2}\right)^{2}-\beta^{2}+\gamma^{2}+\delta^{2}+b\left(-\frac{b}{2}\right)+c & =0 \\
\frac{b^{2}}{4}-\beta^{2}+\gamma^{2}+\delta^{2}-\frac{b^{2}}{2}+c & =0 \\
\beta^{2}-\gamma^{2}-\delta^{2} & =\frac{4 c-b^{2}}{4},
\end{aligned}
$$

where

$$
\beta, \gamma, \delta \in \mathbb{R}
$$


hence, $x=(-b / 2+\beta i+\gamma j+\delta k)$, where $\beta^{2}-\gamma^{2}-\delta^{2}=\left(4 c-b^{2}\right) / 4$ and $\beta, \gamma, \delta \in \mathbb{R}$.

Example. Consider $x^{2}-x+1=0, b=-1, c=1,4 c-b^{2}=3$,

$$
\begin{aligned}
x & =\frac{1}{2}(-b+\beta i+\gamma j+\delta k), \\
\beta^{2}-\gamma^{2}-\delta^{2} & =\frac{4 c-b^{2}}{4}=\frac{3}{4}
\end{aligned}
$$

this implies $\beta^{2}=\frac{3}{4}+\gamma^{2}+\delta^{2}$.

It is satisfied for all choices of $\gamma, \delta \in \mathbb{R}$; let $\gamma=1, \delta=1$; then $\beta=\sqrt{11} / 2$, and

$$
x=\left(\frac{1}{2}+\frac{\sqrt{11}}{2} i+j+k\right) .
$$

Corollary 3. The quadratic equation $x^{2}+b x+c=0$ has infinitely many solutions for $b^{2}<4 c$ and $\beta \neq 0$, in any case. If $\beta=0$, then $4 c-b^{2}=-4\left(\gamma^{2}+\delta^{2}\right)$ is absurd.

Theorem 4. If $b, c \in \mathbb{R}$ and $b^{2}<4 c$, then $x=(-b / 2+\beta i+\gamma j+$ $\delta k$ ) is a fixed point of a class of functions of the form $f(x)=$ $x^{2}+(b+1) x+c$, where $\beta^{2}-\gamma^{2}-\delta^{2}=\left(4 c-b^{2}\right) / 4$ and $\beta, \gamma, \delta \in \mathbb{R}$.

Proof. It is enough to give a new relation $f(x)=g(x)+x$, where $g(x)=x^{2}+b x+c$; then, the existence of fixed points for $f(x)$ is equivalent to the solutions of $g(x)$. Then, the required result is immediate from Proposition 2.

Proposition 5. If $b, c \in \mathbb{R}$ and $b^{2} \geq 4 c$, then $x=(-b / 2+\beta i+$ $\gamma j+\delta k)$ is a root of $x^{2}+b x+c=0$, where $\beta^{2}-\gamma^{2}-\delta^{2}=\left(4 c-b^{2}\right) / 4$ and $\beta, \gamma, \delta \in \mathbb{R}$.

Proof. Consider $b, c \in \mathbb{R}$ and $b^{2} \geq 4 c$.

Put $x=\alpha+\beta i+\gamma j+\delta k$ in $x^{2}+b x+c=0$ :

$$
\begin{aligned}
& (\alpha+\beta i+\gamma j+\delta k)^{2}+b(\alpha+\beta i+\gamma j+\delta k)+c=0 \\
& \alpha^{2}-\beta^{2}+\gamma^{2}+\delta^{2}+2 \alpha \beta i+2 \alpha \gamma j+2 \alpha \delta k+b \alpha+b \beta i \\
& \quad+b \gamma j+b \delta k+c=0 \\
& \alpha^{2}-\beta^{2}+\gamma^{2}+\delta^{2}+b \alpha+c=0 \\
& 2 \alpha \beta+b \beta=0 \\
& 2 \alpha \gamma+b \gamma=0 \\
& 2 \alpha \delta+b \delta=0 \\
& \beta \neq 0 \\
& \text { so } 2 \alpha+b=0 \Longrightarrow \alpha=-\frac{b}{2} ;
\end{aligned}
$$

put $\alpha=-b / 2$; hence,

$$
\begin{aligned}
\left(-\frac{b}{2}\right)^{2}-\beta^{2}+\gamma^{2}+\delta^{2}+b\left(-\frac{b}{2}\right)+c & =0 \\
\frac{b^{2}}{4}-\beta^{2}+\gamma^{2}+\delta^{2}-\frac{b^{2}}{2}+c & =0 \\
-\beta^{2}+\gamma^{2}+\delta^{2} & =\frac{b^{2}-4 c}{4}
\end{aligned}
$$

where

$$
\beta, \gamma, \delta \in \mathbb{R}
$$

hence, $x=(-b / 2+\beta i+\gamma j+\delta k)$, where $b^{2} \geq 4 c$ and $-\beta^{2}+$ $\gamma^{2}+\delta^{2}=b^{2}-4 c$.

Example 6. Consider $x^{2}-2 x+1=0$; here, $b^{2}-4 c=4-4=0$ and $\beta^{2}=\gamma^{2}+\delta^{2}$; we can choose infinitely many values of $\beta, \gamma, \delta \in \mathbb{R}$; let $\gamma=1=\delta$; then, $\beta=\sqrt{2}$; then $x=(-b / 2+$ $\beta i+\gamma j+\delta k)=(1 / 2+\sqrt{2} i+j+k)$.

Corollary 7. It has infinitely many solutions for $b^{2}-4 c \geq 0$.

Theorem 8. $x=(-b / 2+\beta i+\gamma j+\delta k)$ with $b, c \in \mathbb{R}$ and $b^{2} \geq 4 c$, where $\beta^{2}-\gamma^{2}-\delta^{2}=\left(4 c-b^{2}\right) / 4$ and $\beta, \gamma, \delta \in \mathbb{R}$, is a fixed point of a class of functions of the form $f(x)=x^{2}+(b+1) x+c$, where $\beta^{2}-\gamma^{2}-\delta^{2}=\left(4 c-b^{2}\right) / 4$ and $\beta, \gamma, \delta \in \mathbb{R}$.

Proof. Again, it is enough to use relation $f(x)=g(x)+x$, where $g(x)=x^{2}+b x+c$; then, the existence of fixed points for $f(x)$ is equivalent to the solutions of $g(x)$. Then, the required result is immediate from Proposition 5.

Proposition 9. If $b \in \mathbb{R}$ and $c \notin \mathbb{R}$, then $x=(-b / 2 \pm$ $\rho / 2) \mp\left(c_{1} / \rho\right) i \mp\left(c_{2} / \rho\right) j \mp\left(c_{3} / \rho\right) k$ is a root of equation $x^{2}+b x+c=0$, where $c=c_{0}+c_{1} i+c_{2} j+c_{3} k$ and $\beta=-c_{1} /(2 \alpha+b), \gamma=-c_{2} /(2 \alpha+b), \delta=-c_{3} /(2 \alpha+$ b), $\sqrt{\left(b^{2}-4 c_{0} \pm \sqrt{\left(4 c_{0}-b^{2}\right)^{2}+16\left(c_{1}^{2}-c_{2}^{2}-c_{3}^{2}\right)}\right) / 2}=\rho$, and $\operatorname{Re} x=\alpha$

Proof. If $b \in \mathbb{R}$ and $c \notin \mathbb{R}$,

$$
\begin{aligned}
& x=\alpha+\beta i+\gamma j+\delta k, \\
& c=c_{0}+c_{1} i+c_{2} j+c_{3} k, \\
& (\alpha+\beta i+\gamma j+\delta k)^{2}+b(\alpha+\beta i+\gamma j+\delta k)+c_{0}+c_{1} i \\
& \quad+c_{2} j+c_{3} k=0, \\
& \alpha^{2}-\beta^{2}+\gamma^{2}+\delta^{2}+b \alpha=-c_{0}, \\
& 2 \alpha \beta+b \beta=-c_{1},
\end{aligned}
$$




$$
\begin{aligned}
& 2 \alpha \gamma+b \gamma=-c_{2} \text {, } \\
& 2 \alpha \delta+b \delta=-c_{3}, \\
& \beta=-\frac{c_{1}}{2 \alpha+b}, \gamma=-\frac{c_{2}}{2 \alpha+b}, \delta=-\frac{c_{3}}{2 \alpha+b}, \\
& \alpha^{2}-\left(-\frac{c_{1}}{2 \alpha+b}\right)^{2}+\left(-\frac{c_{2}}{2 \alpha+b}\right)^{2}+\left(-\frac{c_{3}}{2 \alpha+b}\right)^{2} \\
& +b \alpha=-c_{0} \text {, } \\
& \alpha^{2}+b \alpha-\frac{c_{1}^{2}}{(2 \alpha+b)^{2}}+\frac{c_{2}^{2}}{(2 \alpha+b)^{2}}+\frac{c_{3}^{2}}{(2 \alpha+b)^{2}}=-c_{0}, \\
& \left(\alpha+\frac{b}{2}\right)^{2}-\frac{c_{1}^{2}}{(2 \alpha+b)^{2}}+\frac{c_{2}^{2}}{(2 \alpha+b)^{2}} \\
& +\frac{c_{3}^{2}}{(2 \alpha+b)^{2}}=-c_{0}+\frac{b^{2}}{4} \\
& \frac{(2 \alpha+b)^{2}}{4}(2 \alpha+b)^{2}-c_{1}^{2}+c_{2}^{2} \\
& +c_{3}^{2}=(2 \alpha+b)^{2}\left(\frac{b^{2}}{4}-c_{0}\right), \\
& (2 \alpha+b)^{4}-4\left(c_{1}^{2}-c_{2}^{2}-c_{3}^{2}\right)=(2 \alpha+b)^{2}\left(b^{2}-4 c_{0}\right), \\
& (2 \alpha+b)^{4}+\left(4 c_{0}-b^{2}\right)(2 \alpha+b)^{2} \\
& -4\left(c_{1}^{2}-c_{2}^{2}-c_{3}^{2}\right)=0 .
\end{aligned}
$$

It is quadratic in $(2 \alpha+b)^{2}$; hence,

$$
\begin{aligned}
& (2 \alpha+b)^{2} \\
& \quad=\frac{-\left(4 c_{0}-b^{2}\right) \pm \sqrt{\left(4 c_{0}-b^{2}\right)^{2}+16\left(c_{1}^{2}-c_{2}^{2}-c_{3}^{2}\right)}}{2}
\end{aligned}
$$

$2 \alpha+b$

$$
= \pm \sqrt{\frac{b^{2}-4 c_{0} \pm \sqrt{\left(4 c_{0}-b^{2}\right)^{2}+16\left(c_{1}^{2}-c_{2}^{2}-c_{3}^{2}\right)}}{2}}
$$

let

$$
\sqrt{\frac{b^{2}-4 c_{0} \pm \sqrt{\left(4 c_{0}-b^{2}\right)^{2}+16\left(c_{1}^{2}-c_{2}^{2}-c_{3}^{2}\right)}}{2}}=\rho
$$

$2 \alpha+b= \pm \rho$,

$2 \alpha= \pm \rho-b$,

$\alpha= \pm \frac{\rho}{2}-\frac{b}{2}$

$x=\alpha+\beta i+\gamma j+\delta k$,

$$
\begin{aligned}
& x=\alpha-\frac{c_{1}}{2 \alpha+b} i-\frac{c_{2}}{2 \alpha+b} j-\frac{c_{3}}{2 \alpha+b} k, \\
& x=\left(-\frac{b}{2} \pm \frac{\rho}{2}\right)-\frac{c_{1}}{ \pm \rho} i-\frac{c_{2}}{ \pm \rho} j-\frac{c_{3}}{ \pm \rho} k \\
& x=\left(-\frac{b}{2} \pm \frac{\rho}{2}\right) \mp \frac{c_{1}}{\rho} i \mp \frac{c_{2}}{\rho} j \mp \frac{c_{3}}{\rho} k .
\end{aligned}
$$

Example 10. One has $x^{2}+3 x+(2+\sqrt{2} i+j+k)=0$ with $b^{2}-4 c_{0}=1>0$ and $\rho=1 / \sqrt{2}$; then $x=(-3 / 2 \pm 1 / 2 \sqrt{2}) \mp$ $2 i \mp \sqrt{2} j \mp \sqrt{2} k$.

Theorem 11. If $x=(-b / 2 \pm \rho / 2) \mp\left(c_{1} / \rho\right) i \mp\left(c_{2} / \rho\right) j \mp$ $c_{3} / \rho$ is a fixed point of functions $x^{2}+(b+1) x+c$, where $c=c_{0}+c_{1} i+c_{2} j+c_{3} k, b \in \mathbb{R}$, and $c \notin \mathbb{R}$ and $\beta=-c_{1} /(2 \alpha+b), \gamma=-c_{2} /(2 \alpha+b), \delta=-c_{3} /(2 \alpha+$ b), $\sqrt{\left(b^{2}-4 c_{0} \pm \sqrt{\left(4 c_{0}-b^{2}\right)^{2}+16\left(c_{1}^{2}-c_{2}^{2}-c_{3}^{2}\right)}\right) / 2}=\rho$, and $\operatorname{Re} x=\alpha$.

Proof. The proof follows as a simple result from Proposition 9 using the same argument as given in previous theorems.

2.1. Open Question and Discussion. We obtained some fixed point results relating to a general quadratic polynomial in algebra of split quaternions. We however remark that if we take $x^{2}+b x+c=0$ with $b, c \notin \mathbb{R}$ and $y^{2}+b y+\dot{c}=0$ with

$$
\begin{aligned}
& y=x+\frac{\operatorname{Re} b}{2}=x+\frac{b_{0}}{2}, \\
& \dot{b}=b-\operatorname{Re} b=b_{1} i+b_{2} j+b_{3} k, \\
& \dot{c}=c-\frac{\operatorname{Re} b}{2}\left(b-\frac{\operatorname{Re} b}{2}\right)=c-\frac{b_{0}}{2}\left(b-\frac{b_{0}}{2}\right), \\
& \dot{c}=c-\frac{b_{0}}{4}\left(b_{0}+2 b_{1} i+2 b_{2} j+2 b_{3} k\right),
\end{aligned}
$$

after putting all these values in $y^{2}+\dot{b} y+\dot{c}=0$, we get $x^{2}+$ $b x+c=0$. Solution of the quadratic equation $y^{2}+b y+\dot{c}=0$ also satisfies $y^{2}-J y+K=0$ with $K=\bar{y} y \geq 0$ for $\beta^{2}>\gamma^{2}+\delta^{2}$ and $J=y+\bar{y} \in \mathbb{R}$. Hence,

$$
\begin{aligned}
(\dot{b}+J) y+(\dot{c}-K) & =0, \\
y & =-(\dot{b}+J)^{-1}(\dot{c}-K)
\end{aligned}
$$

$J \in \mathbb{R}, \hat{b}=b-\operatorname{Re} b \notin \mathbb{R}, \hat{b}+J \neq 0 ;$

$K=y \bar{y}$ implies $K^{2}-K\left(B+J^{2}\right)+E=0$, where $B=\hat{b} \bar{b}+\dot{c}+\bar{c}$, $|c|^{2}=E \in \mathbb{R}$.

$J=y+\bar{y}$ implies $J^{3}+J(\dot{B}-2 K)+D=0$, where $B=-\dot{b} \bar{b}+\dot{c}+$ $\dot{c}, D=\hat{b} \bar{c},+\hat{b} \dot{c}$. Taking the above remark into consideration, we leave this case as an open problem, where we have $b, c \notin \mathbb{R}$. 


\section{Competing Interests}

The authors declare that they have no competing interests.

\section{Authors' Contributions}

All authors contributed equally to the writing of this paper. All authors read and approved the final paper.

\section{Acknowledgments}

This study was supported by research funds from Dong-A University.

\section{References}

[1] Y. Alagöz, K. H. Oral, and S. Yüce, "Split quaternion matrices," Miskolc Mathematical Notes, vol. 13, no. 2, pp. 223-232, 2012.

[2] M. Özdemir, "The roots of a split quaternion," Applied Mathematics Letters, vol. 22, no. 2, pp. 258-263, 2009.

[3] J. H. Conway and D. A. Smith, "On quaternions and octonions: their geometry, arithmetic, and symmetry," Bulletin of the American Mathematical Society, vol. 42, pp. 229-243, 2005.

[4] L. Kula and Y. Yayli, "Split quaternions and rotations in semi Euclidean space," Journal of the Korean Mathematical Society, vol. 44, no. 6, pp. 1313-1327, 2007.

[5] M. Erdoğdu and M. Özdemir, "On eigenvalues of split quaternion matrices," Advances in Applied Clifford Algebras, vol. 23, no. 3, pp. 615-623, 2013.

[6] M. Özdemir and A. A. Ergin, "Some geometric applications of split quaternions," in Proceedings of the 16th International Conference of Jangjeon Mathematical Society, pp. 108-115, 2005.

[7] M. Özdemir and A. A. Ergin, "Rotations with unit timelike quaternions in Minkowski 3-space," Journal of Geometry and Physics, vol. 56, no. 2, pp. 322-336, 2006.

[8] C. B. John, "The octonions," Bulletin of the American Mathematical Society, vol. 39, pp. 145-205, 2002.

[9] C. J. Miguel and R. Serodio, "On the structure of quaternion rings over $\mathbb{Z}_{p}$," International Journal of Algebra, vol. 5, no. 27, pp. 1313-1325, 2011.

[10] F. Zhang, "Quaternions and matrices of quaternions," Linear Algebra and Its Applications, vol. 251, pp. 21-57, 1997.

[11] M. Aristiou and A. Demetre, "Idempotent elements in quaternion rings over $\mathbb{Z}_{p}$," International Journal of Algebra, vol. 6, no. 27, pp. 249-254, 2012. 


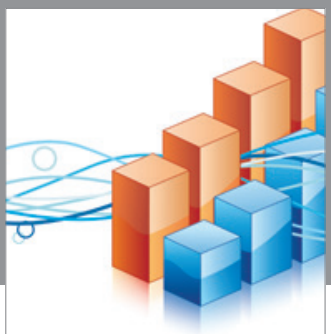

Advances in

Operations Research

vatem alat4

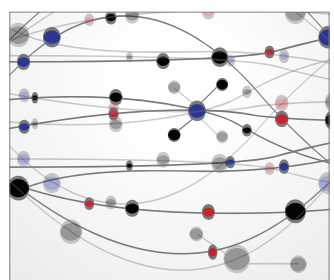

\section{The Scientific} World Journal
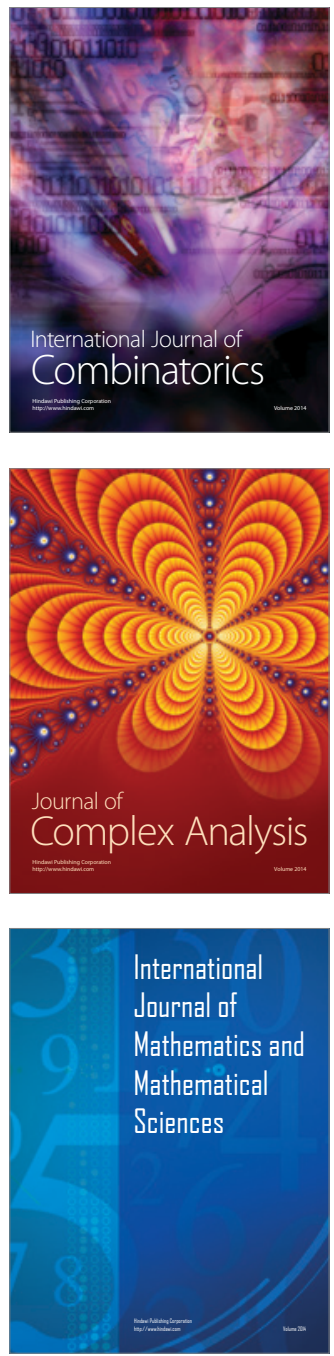
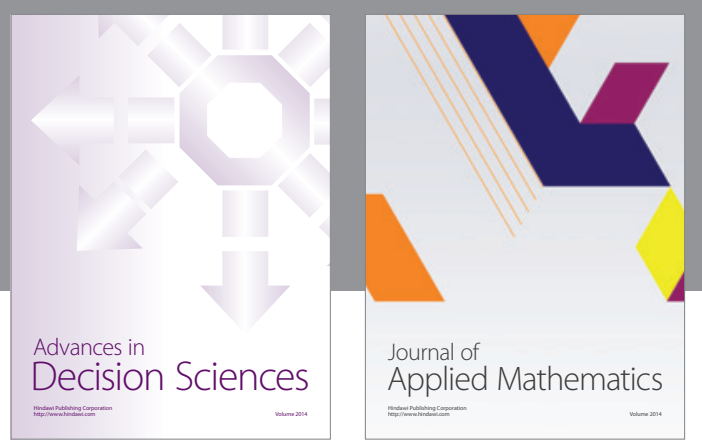

Algebra

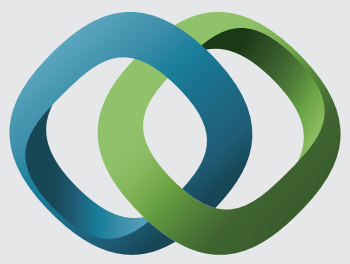

\section{Hindawi}

Submit your manuscripts at

http://www.hindawi.com
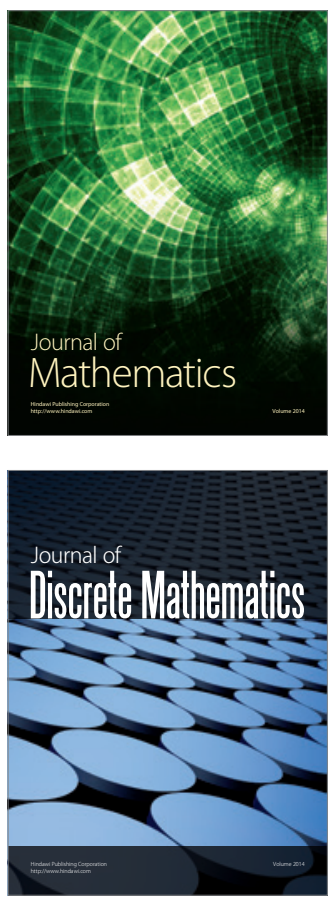

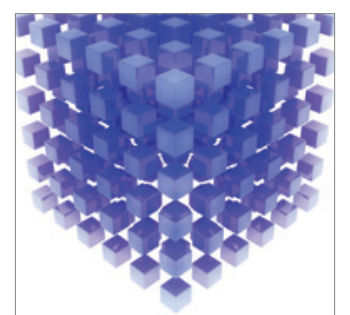

Mathematical Problems in Engineering
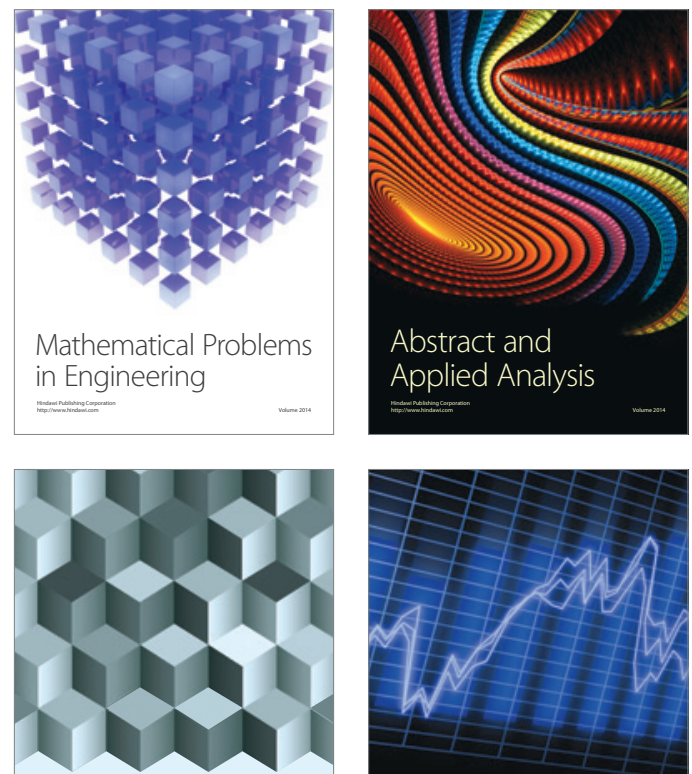

Journal of

Function Spaces

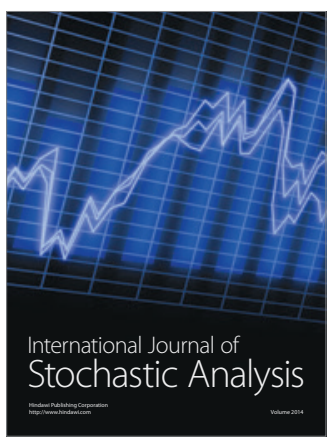

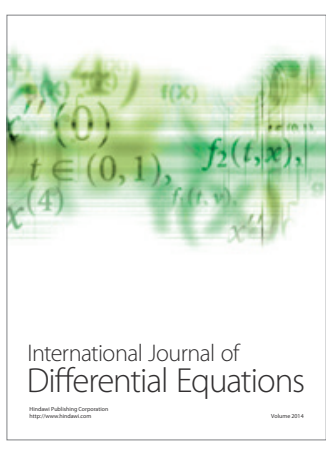
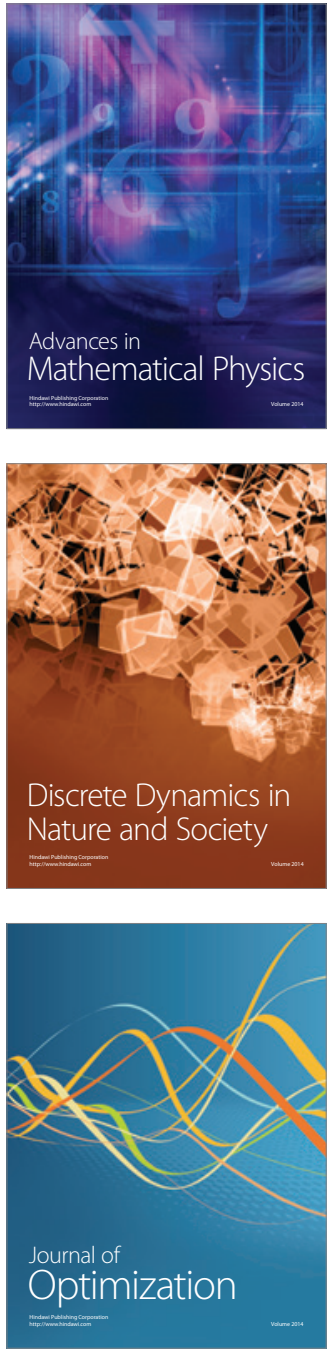